\title{
Aggressive orbital rhabdomyosarcoma in adulthood: A case report in a public hospital in Damascus, Syria
}

\author{
Tagrid Younes Ahmad, Hasan Nabil Al Houri ${ }^{1}$, Abdullah Nabil Al Houri², Nagham Younes Ahmad ${ }^{3}$
}

Department of Internal Medicine, Tishreen hospital, ${ }^{3}$ Department of Ophthalmology, Yusuf Al-Azma Hospital, ${ }^{1}$ Department of Internal Medicine, Al Assad University Hospital and Al Mouwasat University Hospital, Damascus, Syria, ${ }^{2}$ Department of Internal Medicine, University of Jordan, Amman, Jordan

\begin{tabular}{|c|}
\hline Access this article online \\
\hline Website: www.avicennajmed.com \\
\hline DOI: 10.4103/ajm.AJM_62_18 \\
\hline Quick Response Code: \\
\hline
\end{tabular}

\begin{abstract}
Orbital rhabdomyosarcoma (RMS) is a highly malignant tumor that originates mainly from mesenchymal tissue. It is considered a rare childhood malignancy; therefore, presentation in adulthood is scarce. In this case report, a 36-year-old male patient presented with a rapidly progressive RMS of the left orbit. The patient was treated in a different institution with radiochemotherapy with good response. However, relapse occurred after 2 months of completing the course, as rapid growing proptosis. Computed tomography scan revealed a lesion in the left orbit with invasion of the left ethmoid cells and the left maxillary sinus. Exenteration of the left orbit with the left partial maxillectomy and ethmoidectomy was performed. RMS is a highly aggressive malignancy that should be considered in any orbital mass that progresses rapidly. Surgery should be the first line in treatment. Delay in diagnosis and inappropriate treatment negatively affects the outcome.
\end{abstract}

Key words: Adulthood, oncology, ophthalmology, orbital rhabdomyosarcoma

\section{INTRODUCTION}

Soft-tissue sarcomas form $<1 \%$ of all solid tumor malignancies in adults. ${ }^{[1]}$ Out of all soft-tissue sarcomas, only $3 \%$ are rhabdomyosarcomas (RMS). ${ }^{[1]}$ Therefore, it is considered a rare malignancy in adulthood. ${ }^{[1]}$ The most common affected sites are head and neck $(35 \%-40 \%)$ followed by the genitourinary tract and the extremities. Orbital RMS forms only $9 \%$ of all cases in the head and neck. ${ }^{[2]}$

The average age of diagnosis of orbital RMS is $7-8$ years. The tumor has been reported in adults and infants. ${ }^{[3]}$ Its incidence is about four to seven cases per million (approximately 250 cases in children and adolescents aged 15 years or younger) ${ }^{[4]}$ In this case, we report a rare case of aggressive orbital RMS in adulthood.

\section{CASE REPORT}

A 36-year-old male patient was initially seen by an ophthalmologist in Damascus Hospital, with a history of

Address for correspondence: Dr. Hasan Nabil Al Houri, Department of Internal Medicine, Al Assad University Hospital and Al Mouwasat University Hospital, Damascus, Syria. E-mail: hasan94@outlook.sa epiphora (watery eye) along with a reddish mass arising from the conjunctiva of the left lower eyelid. The patient was diagnosed with preseptal cellulitis and was treated orally with an antibiotic, amoxicillin/clavulanic acid (augmentin), $1 \mathrm{~g}$, for 10 days without any benefit. Eyelid was biopsied and showed capillary hemangioma. Dramatic worsening of symptoms led to performing a new biopsy, and computed tomography (CT) was performed [Figures 1and2]. The biopsy showed a malignant, small round-cell tumor, consistent with orbital RMS, with embryonal type. The diagnosis was made dependent on immunohistochemistry staining, using leukocyte common antigen/Desmin/CD99 (positive of Desmin). The patient was referred to oncology and treated with radiochemotherapy. It included cyclophosphamide, doxorubicin, and vincristine with 25 sets of three-dimensional radiotherapy radiology ( $54 \mathrm{~Gy}$ ). He presented to our institution

This is an open access journal, and articles are distributed under the terms of the Creative Commons Attribution-NonCommercial-ShareAlike 4.0 License, which allows others to remix, tweak, and build upon the work non-commercially, as long as appropriate credit is given and the new creations are licensed under the identical terms.

For reprints contact: reprints@medknow.com

Cite this article as: Ahmad TY, Al Houri HN, Al Houri AN, Ahmad NY. Aggressive orbital rhabdomyosarcoma in adulthood: A case report in a public hospital in Damascus, Syria. Avicenna J Med 2018;8:110-3. 
after 2 months of completing radiochemotherapy course with a history of progressive proptosis of the left eyeball. It was associated with blurred vision, diplopia, and headache.

\section{On physical examination}

The patient had low body mass index, and vital signs were stable. The eyeball was displaced temporally. There was chemosis, yellowish discharge, and restriction of eyeball movement [Figure 3]. Ophthalmologic examination showed visual acuity to be one of ten.

\section{Investigations}

Before surgery, multislice CT scan with contrast of the head and neck showed a soft-tissue mass in the left orbit that extended to the left maxillary sinus and invaded the ethmoid cells [Figures 3and4]. CT scan of chest, abdomen, and pelvis showed a density in the anterior part of right lung. A biopsy of the lung mass was recommended to identify whether it is a metastasis; however, the patient refused.

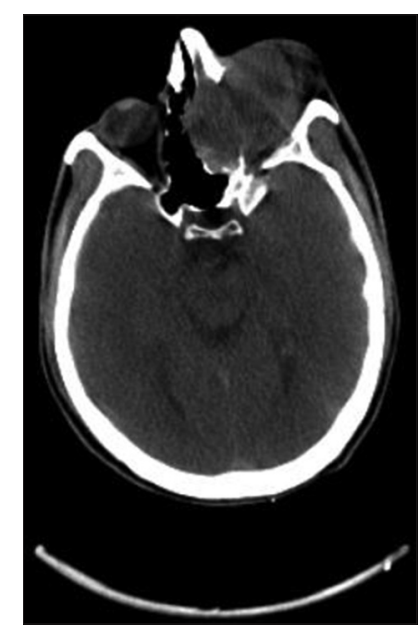

Figure 1: Computed tomography of head. Beiruni Hospital, Damascus, Syria

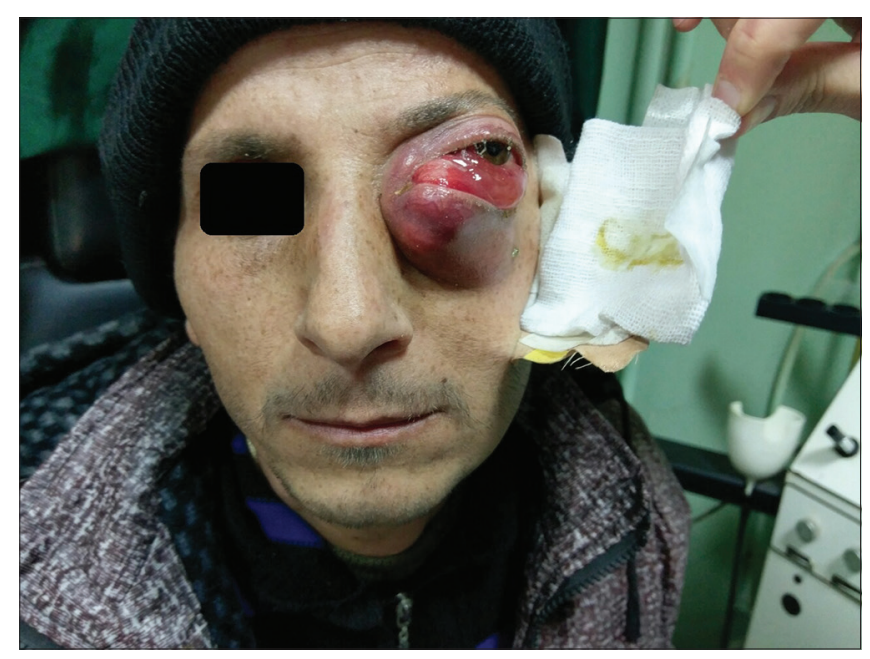

Figure 3: Damascus Hospital, Damascus, Syria

\section{Management}

The patient underwent exenteration of the left orbit, with partial left maxillectomy and left ethmoidectomy. The pathology report showed malignant small, round-cell tumor consistent with orbital RMS, and margins were involved [Figures 5and6]. The tumor measured $5.5 \mathrm{~cm}$ in greatest dimension with invasion of the bone, the left maxillary sinus, and the left ethmoid cells. Ocular globe was intact with no invasion to its compartment identified. The after-surgery staging was not applicable due to not defining whether the mass in the lung was metastatic or not, which may turn it from Stage II to Stage IV.

\section{Follow-up}

The patient has been referred to one oncology-specialized hospital to get adjuvant therapy which included chemotherapy (vincristine + actinomycin D + cyclophosphamide) and 45.0 Gy conventional fractionated irradiation. He was treated as Stage IV guidelines of intergroup RMS Studies, given his lung lesion. Four months after surgery, the patient was doing well. Afterward, the patient had lost to follow-up; he could not be contacted, and he stopped attending his scheduled appointment, and it is not known whether he

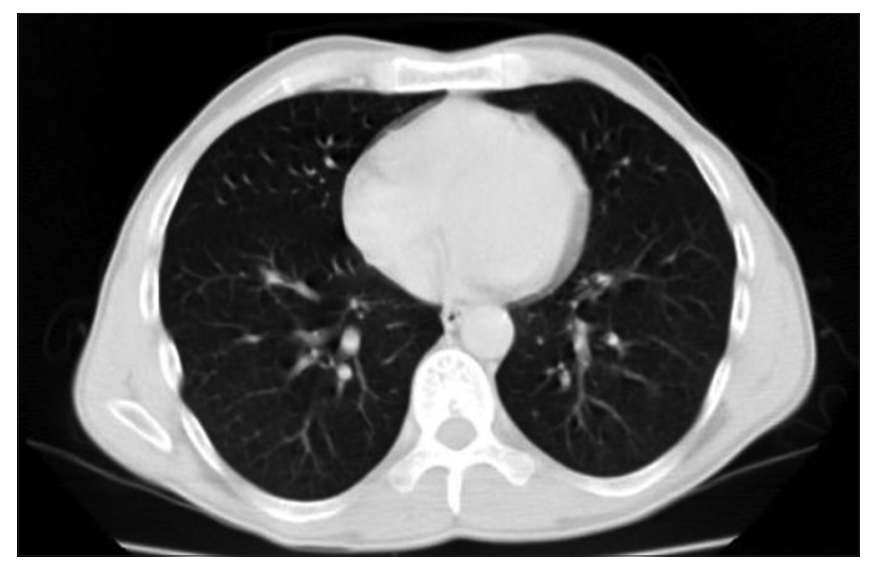

Figure 2: Computed tomography of the chest. Beiruni Hospital, Damascus, Syria

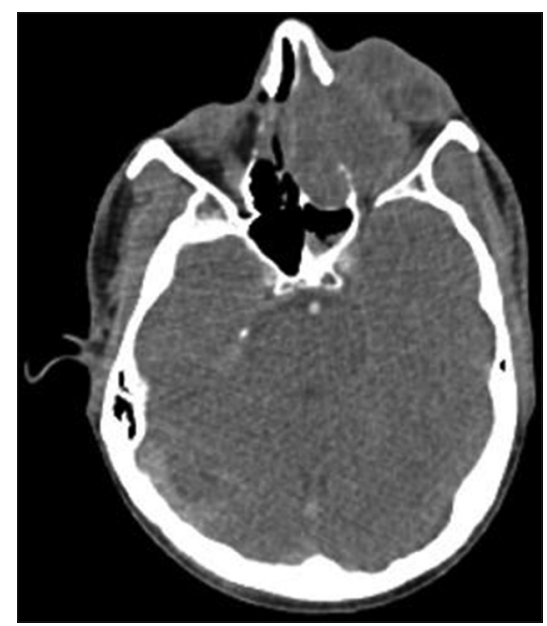

Figure4: Computed tomography scan of the head and neck, Damascus Hospital 


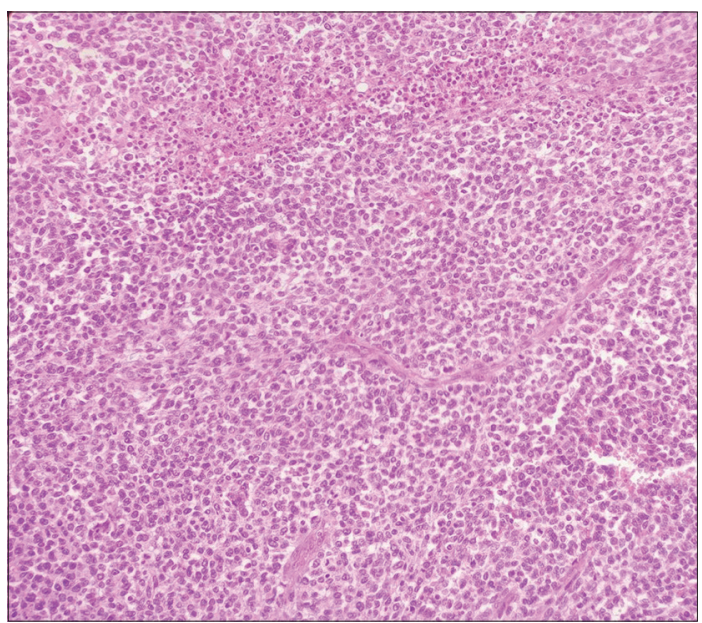

Figure 5: Pathology section. Laboratory of Damascus Hospital

returned to his home in the countryside of Aleppo, where it is still a conflict zone, or whether it was due to health complications.

\section{DISCUSSION}

We report a rare case of primary orbital RMS in adulthood. RMS is considered a disease of young children, which occurs at a mean age of onset of 8 years. ${ }^{[3]}$ RMS is a highly malignant soft-tissue tumor of the skeletal muscle. Histopathologically, RMS is divided into three main categories: embryonal, alveolar, and pleomorphic. Medical literature review revealed only few cases of RMS in adulthood. ${ }^{[5-7]}$ Most of the available data are derived from retrospective case series which state that the typical first presentation is proptosis, ${ }^{[5]}$ while other cases describe atypical presentations such as eyelid nodule. ${ }^{[8]}$ At onset, the patient was misdiagnosed with an inflammatory lesion, then a tumor of vascular origin. However, unresponsiveness to antibiotics and the dramatic clinical progression led to further histopathological study that finally established the diagnosis of orbital RMS. This point raises the importance of considering orbital RMS in the differential diagnosis of any orbital mass that progresses in a similar pattern. Other differential diagnosis may include orbital cellulitis, lymphangioma, hemangioma, metastasis to orbit, lymphoma, dermoid cyst, and chalazion. Awareness of both typical and atypical presentations of orbital RMS could ensure early detection and initiation of appropriate treatment. Nonetheless, it is essential to note that the majority of RMS tumors are not encapsulated, putting orbital contents at risk. ${ }^{[9]}$ Orbital RMS has high tendency to invade the surrounding bone and to extend intracranially, especially in the adult group and even after treatment with radiochemotherapy. ${ }^{[5]}$ Recurrence or metastatic spread usually occur within the first 3 years after treatment, and the most common site of metastasis is the lung. ${ }^{[5,10]}$ Regarding management, there is

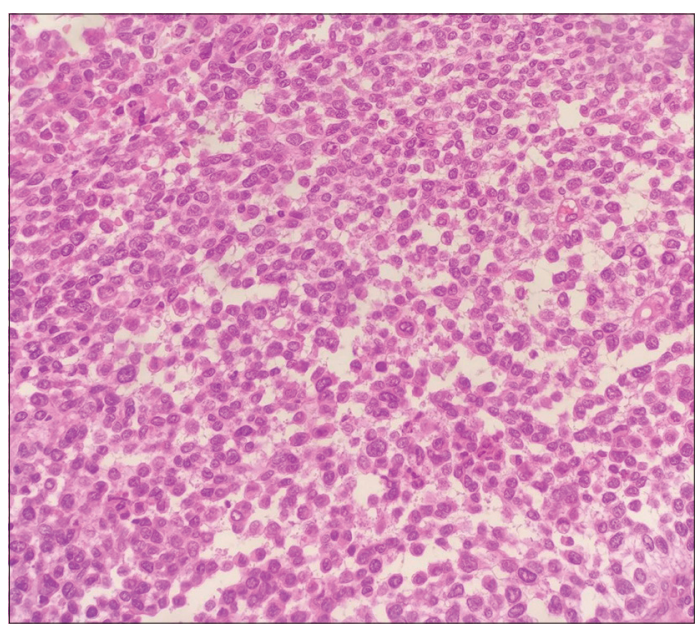

Figure 6: Pathology section. Damascus Hospital

rarity of information available on this topic, and treatment modalities were mainly derived from guidelines dedicated to the management of children with RMS, which was expanded widely to adult group. Historically, orbital RMS was initially treated by exenteration. ${ }^{[9]}$ However, since the early seventies, guidelines were introduced by several study groups, and they recommended the combination of surgery along with adjuvant chemotherapy and radiotherapy according to the stage of the disease. Since then, overall survival improved to $90 \%{ }^{[10]}$ The patient first had courses of radiochemotherapy, but after relapse, the patient underwent exenteration of the left orbit with left partial maxillectomy and ethmoidectomy.

\section{CONCLUSION}

RMS is a highly aggressive malignancy that should be considered in any orbital mass that progresses rapidly. Surgery should be the first line in treatment. Delay in diagnosis and inappropriate treatment negatively affect the outcome.

\section{Declaration of patient consent}

The authors certify that they have obtained all appropriate patient consent forms. In the form, the patient has given his consent for his images and other clinical information to be reported in the journal. The patient understands that name and initials will not be published and due efforts will be made to conceal identity, but anonymity cannot be guaranteed.

Financial support and sponsorship Nil.

\section{Conflicts of interest}

There are no conflicts of interest. 


\section{REFERENCES}

1. Ferrari A, Dileo P, Casanova M, Bertulli R, Meazza C, Gandola L, et al. Rhabdomyosarcoma in adults. A retrospective analysis of 171 patients treated at a single institution. Cancer 2003;98:571-80.

2. Pathology-Rhabdomyosarcoma. Available from: http://www.emedicine. medscape.com/article/873546-overview\#a1.[Last accessed on 2018 Jan 1].

3. McDonough RL, Leib ML. Atypical presentation of orbital rhabdomyosarcoma: A report of two cases. J Pediatr Ophthalmol Strabismus 1995;32:262-5.

4. Young JLJr., Ries LG, Silverberg E, Horm JW, Miller RW. Cancer incidence, survival, and mortality for children younger than age 15 years. Cancer 1986;58:598-602.
5. Kaliaperumal S, Tiroumal S, Rao V. Orbital rhabdomyosarcoma: A case series. Indian J Cancer 2007;44:104-7.

6. Lavaju P, Das H, Malla P, Tiwari A, Sinha A, Upadhyaya P, et al. Orbital rhabdomyosarcoma in an adult. Nepal J Ophthalmol 2010;2:64-7.

7. Garduño-Vieyra L, Hernandez-Da Mota SE, Gonzalez CR, Gamez-Carrillo R. Rhabdomyosarcoma in an elderly patient. A case report. Rev Mex Oftalmol 2017;91:73.

8. Frayer WC, Enterline HT. Embryonal rhabdomyosarcoma of the orbit in children and young adults. AMA Arch Ophthalmol 1959;62:203-10.

9. Ashton N, Morgan G. Embryonal sarcoma and embryonal rhabdomyosarcoma of the orbit. Journal of Clinical Pathology 1965;18:699-714.

10. Jurdy L, Merks JH, Pieters BR, Mourits MP, Kloos RJ, Strackee SD, et al. Orbital rhabdomyosarcomas: A review. Saudi J Ophthalmol 2013;27:167-75. 